\title{
A Method for Analyzing the Radial Distribution of Flexible Ribbon Particles and Cluster in a Fluidized Bed Riser
}

\author{
Kai Wu ${ }^{1,2}$, Bin $\mathrm{Li}^{2}$, Zhulin Yuan' \\ ${ }^{1}$ Key Laboratory of Energy Thermal Conversion and Control of Ministry of Education, Southeast University \\ Nanjing 210096, Jiangsu, China \\ 230169014@seu.edu.cn; 101004322@seu.edu.cn \\ ${ }^{2}$ Key Laboratory of Tobacco Processing Technology, Zhengzhou Tobacco Research Institute of CNTC \\ Zhengzhou 450001, Henan, China \\ ztrilibin@163.com
}

\begin{abstract}
Many biomass materials, such as herbage, tobacco leaf and seaweed are chopped into small strips and usually dried by fluidized bed dryer. These Flexible ribbon particles in the fluidized bed riser may have experienced a much more complicated process, such as heat \& mass transfer, mixing and drying. They are easy to hold together due to the flexibility and the non-uniformity of ribbon shape. Thus, it is important to understand the distribution of this special particle and cluster in the riser. High-speed camera method has always been used in measurement. However, it is difficult to analyze the particle flow due to the overlaps of the flexible ribbon particle in the shot image. So this paper presented a new analyzing method in order to obtain more information of this special gas-solid flow. And the radial concentration distribution of flexible filamentous particle and cluster in gas-solid flow in were experimentally analyzed. It is found that the concentration distribution of the flexible filamentous cluster was completely different from that of the conventional spherical particles.
\end{abstract}

Keywords: Flexible Ribbon Particle, Cluster, Radial Distribution, Multiple Linear Regression.

\section{Introduction}

Fluidized bed processes are widely used in a variety of commercial processes such as combustion of solid fuels, gasification of coal, transport of solid materials, drying of materials, etc. The used granular materials are extensive, including wide range of particle size, different physical properties and shapes. For instance, herbage, tobacco leaf and seaweed are chopped into small strips and usually dried by fluidized bed dryer. These particles are long strip shape with flexibility, usually called flexible ribbon particles[1].

These types of particles are easy to hold together due to the flexibility and the non-uniformity of ribbon shape. So the particles in the gas-solid fluidized bed would exist in the form of a single discrete particle and clusters. It is important to note that this paper also uses the term "cluster", but the physical meaning of flexible ribbon particle cluster is different from the traditional particle cluster due to the special properties of the particle. The hydrodynamic characteristics would impacted by the inhomogeneity of the particle flow, and the performance of fluidized bed is affected by solids mixing, entrainment and heat-mass transfer [2,3]. Thus, it is important to understand the distribution of this special particle and cluster in the riser.

Geng et al.[4] simulated the flow of flexible filamentous particles by Euler-Lagrange method to capture fluidization behavior in a two-dimensional cold bed dryer. The gas flow was defined as continuous phase and the individual flexible filamentous particle was simulated with the chain model. Wang et al.[5] investigated the particle behavior in a bubbling fluidized bed with non-spherical particles using discrete hard sphere method. Li et al. [6] proposed a new algorithm to approximate real particles using multiple overlapping spheres as numerical models. Tao et al. [7] simulated the corn-shaped particles flow in hopper based on a developed DEM model. Zhang et al. [8] investigated the mixing and segregation behavior of biomass particle in fluidized bed experimentally. Although many researchers had done a lot of research on the distribution characteristics of the regular particles[9-13], such as glass beads, quartz sand, relative characteristics study was scarcely concern about the particles of this special flexible ribbon type.

Measurement of the particle concentration distribution in the riser has always been an experimental challenge. Highspeed camera method has always been used in measurement. Because it is a non-contact method, and does not interfere with 
the flow. However, it is difficult to analyze the particle flow due to the overlaps of the flexible ribbon particle in the shot image. So this paper presented a new analyzing method in order to obtain more information of this special gas-solid flow. And the radial concentration distribution of flexible filamentous particle and cluster in gas-solid flow in were experimentally analyzed.

\section{Experimental Setup}

\subsection{Experimental Apparatus}

A cold CFB unit (Fig. 1) was used to study the distribution of flexible ribbon particles and clusters. The system consisted of a riser, a cyclone separator, an air pipe, a feeding device, a fan, a high speed camera and other components. The horizontal section of the riser was circular with diameter of $0.18 \mathrm{~m}$. The height of the riser was $5.2 \mathrm{~m}$. The riser was made up of transparent tempered glass for the convenience of observation and image analyzes of the solid flow in the freeboard of the riser. The air was introduced from the bottom of the riser into the bed by fan. The flexible ribbon particles were fed from the hopper into the riser and then moved upwards with the air. Finally, the particles moved into the cyclone separator, with the flexible ribbon particles separated into the hopper in gas-solid separation process and the air returned to the fan. The upper end of the cyclone separator was connected with the inlet of the inverter fan to form a closed system. In order to simplify the problem, this paper only used the riser as the research object.

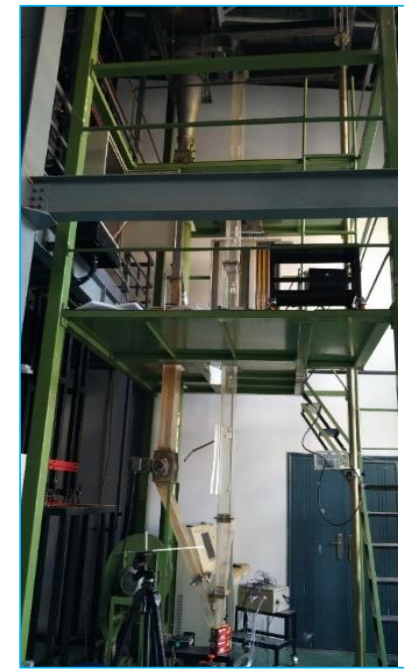

(a)

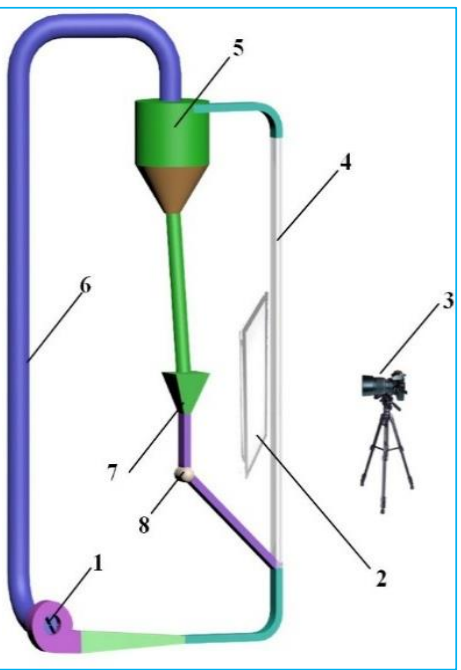

(b)

Fig. 1: Experimental Apparatus. ((a) Photograph of the lab-scale CFB. (b) Functional diagram of the CFB. (1 fan, 2 parallel light source (with Diffuser Panel), 3 high speed camera, 4 riser, 5 cyclone separator, 6 air pipe, 7 hopper, 8 feeder).

\subsection{Measuring System}

A high speed camera with a $50 \mathrm{~mm}$ f/1.4 lens was used for photography with light sensitivity of ISO 100. The exposure duration was set to $100 \mu \mathrm{s}$. The fluidized bed was illuminated from riser back by parallel LED integrated light source, which was then dispersed by parchment paper in the experiments. The measurements were carried out at height of $2.1 \mathrm{~m}-2.4 \mathrm{~m}$.

\subsection{Experimental Materials}

The flexible ribbon particles used in the experiment were flue-cured cut tobacco, as seen in Fig 2(a). The length distribution could be seen in Fig. 2(b). Some degrees of shredding were always produced in biomass cut-tobacco particles in the fluidization cycle, affecting the consistency of the cut-tobacco shape. By making a large number of measurements beforehand, the measurement time of 40 seconds was found to be sufficient and appropriate because the particle feature did not change significantly during this period. In order to ensure the consistency of the flexible ribbon particle shape, cuttobacco was not repeatedly used.

The larger the superficial gas velocity, the stronger the frictional static electricity between the cut-tobacco particles and walls, the greater the changes in moisture content and shredding degree. After comprehensive consideration, the bed 
inventory $\left(M_{\mathrm{s}}\right)$ of $750 \mathrm{~g}$ and the superficial gas velocity $(\mathrm{Ug})$ of $5.52 \mathrm{~m} / \mathrm{s}$ were chosen in this experiments. And two-phase flow was in a dilute phase pneumatic conveying state.

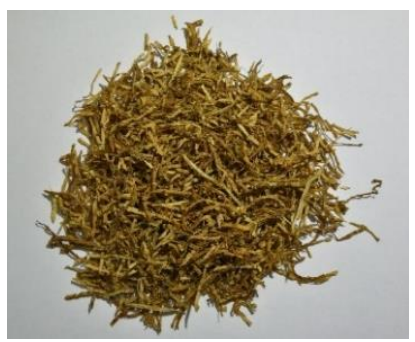

(a)

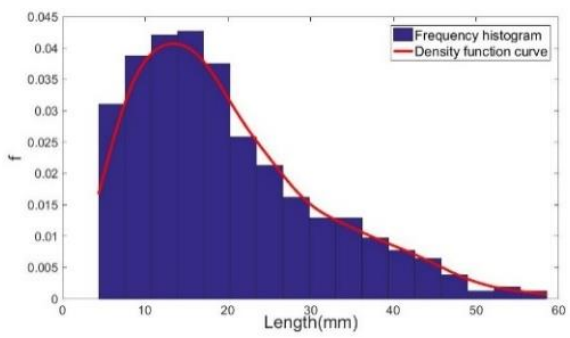

(b)

Fig. 2: Flue-cured cut tobacco. ((a)Flue-cured cut tobacco, (b)The length distribution of cut-tobacco particles).

\section{Analysis Method}

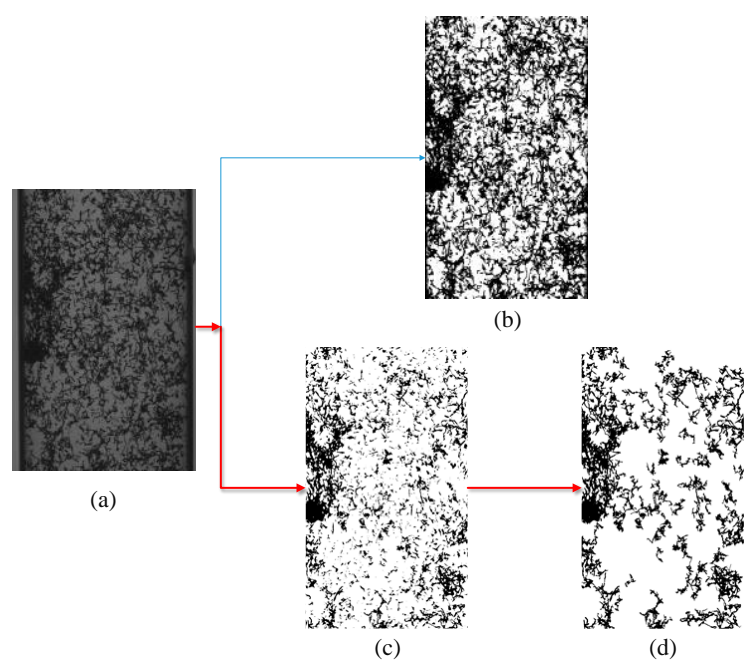

Fig. 3. A transient image of flexible ribbon particles flow in the riser.

(a. Origin image; b. Binarization image; c. Extracted binary image; d. Cluster image).

The instantaneous image of flexible ribbon flow with a bed inventory of $750 \mathrm{~g}$ and a superficial gas velocity of $5.52 \mathrm{~m} / \mathrm{s}$ is shown in Fig. 3 as an example. In order to identify the particle shape from the image background, conventional processing method was often used. First, the instantaneous photos of the flexible ribbon particles (Fig. 3(a)) was taken by the high-speed camera. Then, the picture was binarized directly, as shown in Fig. 3(b). However, it is difficult to analyze the particle flow due to the overlaps of the flexible ribbon particle in the incident light direction of the riser. According to the grayscale analyzes of the image, a cut from the incident light direction was made in this step based on the MATLAB software for optimization of the particle and cluster identification.

\subsection{Extraction of Flexible Ribbon Particle}

When the subject was in a particular space in front of the lens, its imaging on the film was centred between the two diffuse circles, which located at the front and behind the focus point respectively. In this experiments, the focus point was adjusted to located in the riser wall, which was the closest one to the camera. The cut-tobacco particles in the semi-circular column near the camera were imaged in the range of confusion circle, while the cut-tobacco particles in the semi-circular column farther from the camera were imaged outside the confusion circle range. The latter image was blurred on the film, and their corresponding pixels have a higher gray value. By choosing an appropriate threshold, the particles in the semicircle section farther away from the camera can be filtered by filtering. The specific way was as follows.

The focus depth can be simplified in Eq. (1). 


$$
\Delta L_{1}=\frac{F \delta L^{2}}{f^{2}-F \delta L}
$$

where, $\delta$ is diameter of dispersion circle, $(\mathrm{m}) . f$ is Lens focal length, $(\mathrm{mm}) . F$ is aperture value of the lens, $F=f / D . D$ is effective aperture of lens. $L$ is focusing distance, $(\mathrm{m}) . \Delta L_{1}$ is back depth of focus, $(\mathrm{m})$. In the empty bed condition, the gray value of the image was about 0.38 , which ignores the influence of the field angle on the image surface illumination.

According to the Lambert-Beer law, the proportion of light absorbed by the transparent medium is independent of the intensity of the incident light. It is obvious that the image surface illumination $E$ (lx) is proportional to the brightness of the measured surface. So Eq. (2) is obtained.

$$
E\left[\frac{\Delta L_{1} f^{2}}{F L\left(\Delta L_{1}+L\right)}\right]^{2}=\text { const }
$$

$L=1.5 \mathrm{~m}, F=4, f=50 \mathrm{~mm}$ were set in this experiments. The appropriate back depth of focus $\left(\Delta L_{1}\right)$ should be $0.090 \mathrm{~m}$, so the diameter of dispersion circle was $0.0236 \mathrm{~mm}$. Empty bed was used to standardize the results, the surface illumination $E$ at the same location in the image of the empty riser was 0.38 with the back depth of focus $\Delta L_{1}$ of $0.180 \mathrm{~m}$. Thus, the diameter of dispersion circle was obtained to filter out the pixels out of the appropriate gray-scale intensity value. Through the above steps, the flexible ribbon particles in the posterior semi-circular riser can be filtered out.

\subsection{Image Thresholding and the Division of Connected Domains}

In order to identify and extract particles and clusters from the background of images, the appropriate threshold value was needed to convert the processed image into a binary image. The threshold value of 0.7 was chose in present works and the results were illustrated in Fig. 3(c). Fig. 3(c) displayed the binary image after filtering in the incident light direction of the riser. The shape of the particles and clusters in the images after processing was easy to be outlined. Compared to Fig. 3 (b), only about half of the particles were left, and the average gray value of the image was increased significantly.

The binary image shown in Fig. 3(c) contained all particle morphology (single particle and cluster). In order to extract the flexible ribbon clusters, the connected area with the area larger than a certain fixed value in the binary image (Fig. 3(c)) were reserved, while the others were deleted. Finally, Fig. 3(d) was obtained, so that the cluster characteristics of the flexible ribbon particles can be easily analyzed. Thus, the domain area threshold value of 1200 pixels (The image resolution was $979 \times 1696)$ was selected to distinguish the cluster from single particle.

\subsection{Analysis of Concentration Distribution}

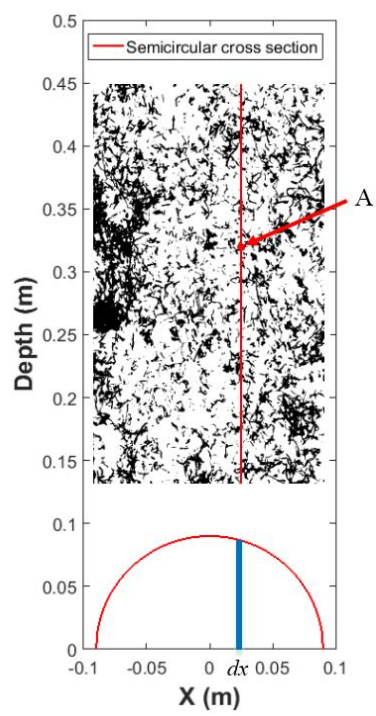

Fig. 4: The distance in the depth direction of the semi-circular section. 
For the processed image (Fig. 3(c), 3(d)), each pixel of the image reflects the particle concentration in the depth direction of the semi-circular section. Depth distance of the semi-circular section was shown in Fig. 4. For example, the gray value of pixel at any point $A$ in the image with the horizontal coordinate $x$ reflected the particle concentration at the half depth $(y)$ of the riser at the horizontal cross-section of $x$. It is assumed that the average concentration of particles in the riser has a certain relationship with the radial position, and it is set to $f(r / R) . R$ is $0.09 \mathrm{~m}$, which is the radius of the riser.

It is assumed that the average pixel gray value in the horizontal direction of the image is $\mathrm{g}(x)$. For circular cross-section riser, $f(r / R), g(x)$ must be continuous. So the following formula can be obtained:

$$
\mathrm{g}(X)=\int_{X}^{1} \frac{f(t) t}{\sqrt{t^{2}-X^{2}}} d t \quad(X=x / R, t=r / R)
$$

Assume that the function $f(t)$ has an $\mathrm{n}$-th order derivative over some closed interval that containing $\mathrm{x}_{0}$. According to Taylor's formula, for any point $\mathrm{x}$ on the closed interval, the following holds,

$$
f(r / R)=f(t)=\frac{f\left(x_{0}\right)}{0 !}+\frac{f^{\prime}\left(x_{0}\right)}{1 !}\left(x-x_{0}\right)+\frac{f^{\prime \prime}\left(x_{0}\right)}{2 !}\left(x-x_{0}\right)^{2}+\mathrm{L}+\frac{f^{(n)}\left(x_{0}\right)}{n !}\left(x-x_{0}\right)^{n}+R_{n}(x)
$$

Let $x_{0}$ be 0 , the above formula can be written as,

$$
f(r / R)=f(t)=a_{0}+a_{1} t+a_{2} t^{2}+\mathrm{L}
$$

\begin{tabular}{|c|c|c|c|c|c|}
\hline & $g(X)=\left(a_{0}, a_{1}, a_{2},, \mathrm{~L} a_{n}\right)$ & $\left(\begin{array}{c}f\left(X_{0}, t\right) \\
f\left(X_{0}, t^{2}\right) \\
\mathrm{M} \\
f\left(X_{0}, t^{n}\right)\end{array}\right.$ & $\begin{array}{c}f\left(X_{1}, t\right) \\
f\left(X_{1}, t^{2}\right) \\
\mathrm{M} \\
f\left(X_{1}, t^{n}\right)\end{array}$ & $\left.\begin{array}{cc}\mathrm{L} & f\left(X_{n}, t\right) \\
\mathrm{L} & f\left(X_{n}, t^{2}\right) \\
\mathrm{O} & \mathrm{M} \\
\mathrm{L} & f\left(X_{n}, t^{n}\right)\end{array}\right)$ & (6) \\
\hline
\end{tabular}

Then,

The radial profile concentration distribution of the flexible ribbon particles $(f(t))$ can be obtained by multiple linear regression. For cluster,

$$
\mathrm{g}_{c}(X)=\int_{X}^{1} \frac{f_{c}(t) t}{\sqrt{t^{2}-X^{2}}} d t \quad(X=x / R, t=r / R)
$$

Similarly, the radial profile distribution of cluster $\left(f_{c}(t)\right)$ in a circular section riser can be obtained.

\section{Result}

\subsection{Distribution of Particle and Cluster Concentration in the Image}

1000 images were selected as the sample under the same operating condition, and the time interval was $0.03 \mathrm{~s}$. Through mathematical statistics, the average particle concentration $\mathrm{g}(x)$ and cluster concentration $\mathrm{g}_{\mathrm{c}}(x)$ in the horizontal direction of the image could be obtained, as shown in Fig. 5. 


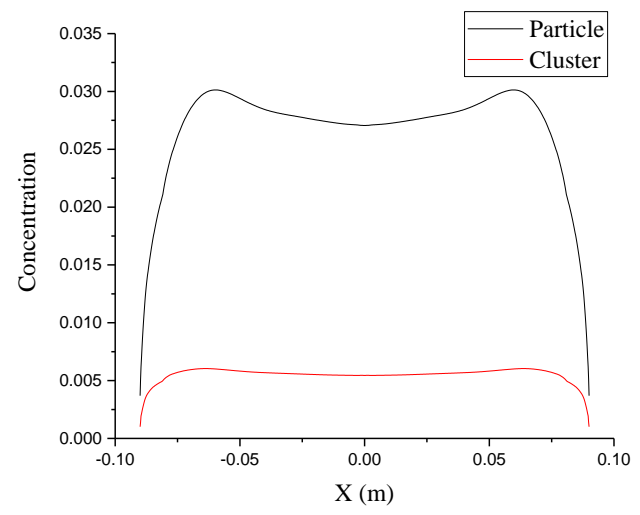

Fig. 5: The average particle and cluster concentration in the horizontal direction of the image.

In theory, the concentration at the neighboring wall should be zero, that is, the average concentration of particles and cluster at $|\mathrm{x}|=0.09 \mathrm{~m}$ should be zero. However, the actual object width represented by one pixel was $0.0000184 \mathrm{~m}$ in this paper. which was not an extreme boundary. The boundary of the image was not the boundary in the limit sense. Therefore, the two ends of the image were filled with two columns of blank pixels, and its influence on the calculation results could be ignored. The result reached the theoretical limit of 0 at the neighboring wall.

As can be seen from Fig. 5, the average concentration distribution of particles and clusters in the horizontal direction of the image was not uniform. The Fig. 5 showed a saddle-like distribution of the average particle concentration in the horizontal direction. From two horizontal ends of the image to the center, the particle concentration first increased sharply from 0 and reached a maximum value of 0.0301 at $|\mathrm{x}|$ of $0.06 \mathrm{~m}$, then descended slowly until it reached the center. The particle concentration at the image center is 0.027 . At the image center, the depth y was the largest, while the particle concentration was the minimum in the vicinity, indicating that the distribution of particles was uneven at the radial direction, and the concentration of particles in the image center was small.

The concentration distribution of particle and particle cluster was completely different. From the horizontal ends of the image to the center, the cluster concentration only in the near edge of the image increased sharply, reaching the maximum at $|\mathrm{x}|$ of $0.064 \mathrm{~m}$ with a maximum of 0.006 . Then, cluster concentration experienced a slight decline until to the center, and the downward trend was very slow. The cluster concentration at the image center was 0.00545 . The cluster concentration in the image center decreased by about $10 \%$ compared to the maximum value at $|\mathrm{x}|$ of $0.064 \mathrm{~m}$. The concentration of clusters is less than the concentration of the particles, which was about one-fifth of the latter. And the coordinate value where the maximum value of the curve occurred was also slightly different. The horizontal coordinate value |xc|, where the maximum concentration of cluster occurred, was greater than that of maximum particle concentration, indicating that the maximum appeared at a position closer to the left and right ends of the image. The closer the point to the edge of the image was, the greater the proportion of the wall portion of the riser. So the concentration of the cluster in the neighboring wall was larger than the concentration of the particles. In other words, the particles cluster is more easily formed in the neighboring wall regions.

\subsection{Radial Distribution of Particle and Cluster Concentration}

The radial distribution of particle concentration and cluster concentration was shown in Fig. 6 . The results indicate that the concentration of the particles in the neighboring wall is greater than the concentration at the center. The particle concentration at the riser center $(r / R=0)$ was 0.015 . As $r / R$ increased, the particle concentration increased slowly. The concentration at $r / R$ of 0.4 was 0.018 . Then, the increasing trend of particle concentration increased. when $r / R$ was 0.6 , the particle concentration was 0.026 . Since then, the particle concentration had increased linearly until $\mathrm{r} / \mathrm{R}$ was 1 and Its slope was 0.08 . Due to the effect of boundary layer, the superficial gas velocity in the neighboring wall region was smaller than that in the central region. The drag force on the particles in the neighboring wall was also smaller. Contrast with the center region, the average particle velocity at the neighboring wall region was also smaller, so the particles was easy to concentrate here, that is, the particle concentration in the neighboring wall region was greater than in the center. The principle of minimization of energy suggested that the gas flow was more likely to flow through the region with less resistance. Therefore, 
the velocity of gas flow in the central region was accelerated, and particles were dilute in the center region of the riser than in the neighboring wall region. It is easier to form cluster in the neighboring wall of riser at high solid concentration.

The concentration of cluster was 0.0049 at the center of riser. As the $\mathrm{r} / \mathrm{R}$ increased, the cluster concentration increased slowly. when $\mathrm{r} / \mathrm{R}$ was 0.3 , the cluster concentration was 0.00335 . The concentration of cluster increased sharply thereafter, with a concentration of 0.0084 at a $\mathrm{r} / \mathrm{R}$ of 0.8 . The increasing tendency of the cluster concentration decreased gradually, reaching a maximum value of 0.0107 at a position close to the wall, and then a slight decrease with a neighboring wall concentration of 0.0103. It can be seen from the comparison that the trend of concentration distribution of particles and cluster was similar except for the region near the neighboring wall. Unlike the particle concentration, the concentration of cluster no longer continued to increase at the neighboring wall. The main reason was that the special material flexible ribbon particles were prone to twisting and entanglement among the particles, so that the interaction between the cluster particles formed was stronger and the separation was more difficult after the formation. The properties of the cluster were completely different from that of the conventional materials, such as glass beads. The cluster formed in this paper was more spherical and more difficult to deform. Since the mass center of the cluster that at the neighboring wall region was usually at a small distance from the wall, so the curve of the cluster concentration decreased slightly at the neighboring wall region.

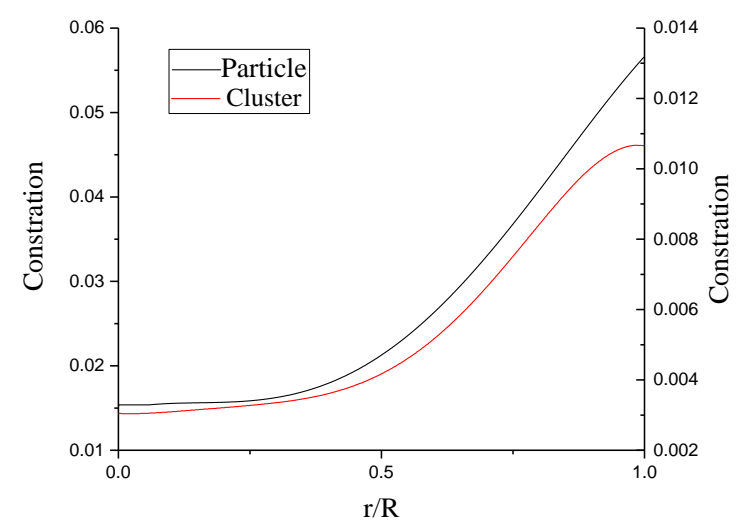

Fig. 6: Radial distribution of particle and cluster concentration.

\section{Conclusions}

Flexible ribbon particles in the fluidized bed riser may have experienced a much more complicated process, such as heat $\&$ mass transfer, mixing and drying. These types of particles are easy to hold together due to the flexibility and the nonuniformity of ribbon shape. Research on flexible ribbon particles in a fluidized bed dryer is significant.

In order to obtain more information on the flow of flexible ribbon particles, this paper presents a new analyzing method. According to the grayscale analyzes of the image, a cut from the incident light direction was made in this step based on the MATLAB software for optimization of the particle and cluster identification. The radial concentration distribution of the flexible ribbon particles $(\mathrm{f}(\mathrm{t})$ ) can be obtained by a series of processes, such as extraction of particle, image thresholding, the division of connected domains, multiple linear regression.

Through mathematical statistics, the average particle and cluster concentration in the horizontal direction of the image was obtained. The average concentration distribution of particles in the horizontal direction of the image was not uniform, and a saddle-like distribution of the average particle concentration occurred.

The concentration of the particles in the neighboring wall is greater than the concentration at the center. It is easier to form cluster in the neighboring wall of riser at high solid concentration. Unlike the particle concentration, the concentration of cluster no longer continued to increase at the neighboring wall. The main reason was that the special material flexible ribbon particles were prone to twisting and entanglement among the particles, so that the interaction between the cluster particles formed was stronger and the separation was more difficult after the formation. Since the mass center of the cluster that at the neighboring wall region was usually at a small distance from the wall, so the curve of the cluster concentration decreased slightly at the neighboring wall region. The presence of clusters made the particle distribution more inhomogeneous and the gas-solid flow in the riser became more complicated. 
It is found that the concentration distribution of the flexible filamentous cluster was completely different from that of the conventional spherical particles. Because the appearance of clusters is unfavorable in many cases, it is of great significance to analyze the radial distribution of clusters and to study the internal mechanism and optimize the equipment performance. The experimental condition was dilute phase pneumatic conveying process in this paper. If the method of dense phase is adopted, it may bring a great error. This will be the focus of the next model study.

\section{Acknowledgement}

Financial support from the National Natural Science Foundation of China (No. 51576046), the Science and Technology Program of Jiangsu, China (No. BY2015070-15) and Key Lab of Tobacco Processing Technology of Zhengzhou Tobacco Research Institute (No. 212016AA0300) are gratefully acknowledged.

\section{Reference}

[1] F. Geng, H. Chai, L. Ma, G. Luo, Y. Li, Z. Yuan, "Simulation of dynamic transport of flexible ribbon particles in a rotary dryer," Powder Technology, vol. 297, pp. 115-125, 2016.

[2] M. Horio, R. Clift, "A note on terminology: 'clusters' and 'agglomerates'," Powder Technology, vol. 70, p. $196,1992$.

[3] M. Horio, H. Ishii, M. Nishimuro, "On the nature of turbulent and fast fluidized beds," Powder Technology, vol. 70, pp. 229-236, 1992.

[4] F. Geng, G. Luo, Y. Li, L. Yuan, Z. Yuan, X. Wu, "Numerical simulation on distribution characteristics of flexible filamentous particles in a fluidized bed dryer," Powder Technology, vol. 267, pp. 322-332, 2014.

[5] M. T. Shah, R. P. Utikar, V. K. Pareek, G. M. Evans, J. B. Joshi, "Computational fluid dynamic modelling of FCC riser: A review," Chemical Engineering Research and Design, vol. 111, pp. 403-448, 2016.

[6] C.-Q. Li, W.-J. Xu, Q.-S. Meng, "Multi-sphere approximation of real particles for DEM simulation based on a modified greedy heuristic algorithm," Powder Technology, vol. 286, pp. 478-487, 2015.

[7] H. Tao, B. Jin, W. Zhong, X. Wang, B. Ren, Y. Zhang, R. Xiao, "Discrete element method modeling of non-spherical granular flow in rectangular hopper," Chemical Engineering and Processing: Process Intensification, vol. 49, pp. 151$158,2010$.

[8] Y. Zhang, B. Jin, W. Zhong, "Experimental investigation on mixing and segregation behavior of biomass particle in fluidized bed," Chemical Engineering and Processing: Process Intensification, vol. 48, pp. 745-754, 2009.

[9] S. Baesch, K. Price, P. Scharfer, L. Francis, W. Schabel, "Influence of the drying conditions on the particle distribution in particle filled polymer films: Experimental validation of predictive drying regime maps," Chemical Engineering and Processing: Process Intensification, vol. 123, pp. 138-147, 2018.

[10] H. Cho, J. Kim, C. Park, K. Lee, M. Kim, I. Moon, "Uneven distribution of particle flow in RFCC reactor riser," Powder Technology, vol. 312, pp. 113-123, 2017.

[11] Y. Wu, L. Peng, L. Qin, M. Wang, J. Gao, X. Lan, "Validation and application of CPFD models in simulating hydrodynamics and reactions in riser reactor with Geldart A particles," Powder Technology, vol. 323, pp. 269-283, 2018.

[12] Q. Wang, T. Niemi, J. Peltola, S. Kallio, H. Yang, J. Lu, L. Wei, "Particle size distribution in CPFD modeling of gassolid flows in a CFB riser," Particuology, vol. 21, pp. 107-117, 2015.

[13] J. Sun, Y. Yan, "Non-intrusive characterisation of particle cluster behaviours in a riser through electrostatic and vibration sensing," Chemical Engineering Journal, vol. 323, pp. 381-395, 2017. 